\title{
Elective Neck Dissection, but Not Adjuvant Radiation Therapy, Improves Survival in Stage I and II Oral Tongue Cancer with Depth of Invasion $>4 \mathrm{~mm}$
}

Justin Mann ${ }^{1}$, Diana Julie ${ }^{2}$, Sean S. Mahase ${ }^{2}$, Debra D'Angelo ${ }^{3}$, Louis Potters ${ }^{4}$, A. Gabriella Wernicke ${ }^{5}$, Bhupesh Parashar ${ }^{4}$

1. Radiation Oncology, Memorial Sloan Kettering Cancer Center, New York, USA 2. Radiation Oncology, NewYorkPresbyterian/Weill Cornell Medical Center, New York, USA 3. Biostatistics and Epidemiology, Weill Cornell Medical Center, New York, USA 4. Radiation Oncology, Zucker School of Medicine at Hofstra/Northwell, New York, USA 5. Radiation Oncology, Weill Cornell Medical Center, New York, USA

Corresponding author: Bhupesh Parashar, bparashar@northwell.edu

\section{Abstract \\ Purpose/objective(s)}

In early-stage, node negative oral tongue cancer, there is limited data supporting tumor depth of invasion (DOI) as an indication for post-operative radiotherapy (PORT) to the primary site. The primary aim of this study is to examine the effect of tumor DOI and PORT on overall survival (OS).

\section{Materials and methods}

The National Cancer Database (NCDB) was used to query patients with AJCC stage I and II oral tongue cancer (2006-2013). Patients were stratified by receipt of PORT, elective neck dissection (ND), and DOI ( $\$ 4$ $\mathrm{mm}$ or $>4 \mathrm{~mm}$ ). Kaplan-Meier analysis was performed to compare OS (using the log-rank test) between PORT versus no-PORT. Multivariable Cox proportional hazards regression model performed to evaluate the independent effect of PORT and neck dissection on OS.

\section{Results}

Among 939 patients, 69.3\% were clinical stage I, 67.4\% received ND, 23.4\% had DOI >4 mm, and $10.4 \%$ received PORT. The addition of PORT did not improve OS with tumor DOI $\leqslant 4 \mathrm{~mm}(\mathrm{p}=0.634)$ or $>4 \mathrm{~mm}(\mathrm{p}=$ $0.816)$. The addition of elective neck dissection improved OS for DOI $>4 \mathrm{~mm}(\mathrm{p}=0.010)$, but not for $\leqslant 4 \mathrm{~mm}$ $(p=0.128)$. On multivariable analysis, ND improved OS if DOI $>4 \mathrm{~mm}$ (HR, 0.37; 95\% CI, 0.17-0.81 [p = .012]), when also controlling for age, sex, PORT status, clinical stage, and pathological stage.

Received 10/14/2019 Review began 10/19/2019 Review ended 11/22/2019 Published 12/04/2019

\section{(c) Copyright 2019}

Mann et al. This is an open access article distributed under the terms of the Creative Commons Attribution License CC-BY 3.0., which permits unrestricted use, distribution, and reproduction in any medium, provided the original author and source are credited.

\section{Conclusion}

Tumor DOI should not be used as a sole indication for PORT in early stage oral tongue cancers. Elective neck dissection at the time of excision of the primary tumor results in higher OS for tumors with DOI $>4 \mathrm{~mm}$.

\section{Categories: Radiation Oncology}

Keywords: depth, invasion, radiation, tongue, cancer, ncdb

\section{Introduction}

Depth of invasion (DOI) is defined as the length measured from the tumor surface to the deepest point of invasive tumor in a paraffin embedded section [1]. The cut-off commonly used to stratify patients into low and high risk is $4 \mathrm{~mm}$ [2]. DOI is an important prognostic factor for nodal metastasis in oral tongue cancer, with increasing DOI associated with nodal involvement and worse prognosis [3-7].

Recent studies demonstrated no benefit to adding radiation therapy (RT) for deeper tumors [5-9]. O'steen et al. retrospectively evaluated the outcomes of 32 patients with stage $\mathrm{N} 0-2 \mathrm{~b}$ oral tongue or floor of mouth cancers with the primary tumor not crossing the midline who underwent PORT. The DOI in $>75 \%$ patients was $>4 \mathrm{~mm},>75 \%$ had positive or close $(<5 \mathrm{~mm}$ ) margins and $38 \%$ had perineural invasion (PNI). RT to contralateral (CL) neck was omitted. At a median follow-up of 5.5 years among patients alive at the end of the study, there were no isolated nodal recurrences despite the majority of tumors possessing of DOI $>4 \mathrm{~mm}$. The authors concluded that the risk of nodal recurrence when omitting CL neck RT was very low if the primary tumor did not cross the midline, irrespective of other risk factors [5].

Rajappa et al. evaluated 375 pT1-2N0 oral tongue cancer patients. The cohort's median age was 49, and 93\% had squamous cell carcinomas, with $37.6 \%$ and $5.87 \%$ possessing PNI and lympho-vascular invasion (LVI), 
respectively. PORT was delivered in $37.6 \%$ of the cohort for PNI/LVI in the majority of cases, and for close margins in the remaining patients. At a mean follow-up of 40.9 months, there was a $18.4 \%$ local recurrence rate, with a 12.7-month mean duration of recurrence. Forty-four percent of the recurrences were salvaged while the remainder developed distant metastasis (DM) or unresectable disease. The two- and five-year overall survival (OS) were $94.5 \%$ and $93.9 \%$, respectively. The patients were further divided into three groups: DOI < mm, 6-10 mm and >10 mm, each of which were categorized into RT vs no-RT groups. Adding RT did not improve OS or disease-free survival (DFS) in any group [8].

A National Cancer Database (NCDB) analysis of 934 patients with pathological T2N0 oral tongue cancers from 2004-2013 was performed to determine whether lesions with >5 mm DOI benefitted from receiving PORT [9]. Six hundred and seventy-seven (72.5\%) patients had surgery alone and 257 (27.5\%) received surgery plus PORT. Thirty-four (13.4\%) received chemotherapy in addition to surgery and PORT. With a median follow-up of 28.4 months +/-10.4 months, the three-year OS was $81.3 \%$. In multivariate analysis (MVA), adding PORT did not improve OS, even for patients with $>5 \mathrm{~mm}$ DOI $(\mathrm{p}=0.769)$.

This study evaluates the potential benefit of PORT in pT1-2N0 (stage I and II) oral tongue cancers with a DOI $>4 \mathrm{~mm}$.

\section{Materials And Methods}

The NCDB is a national oncology database and the data represents $>70 \%$ newly diagnosed cancer cases and $>34$ million historical records [10]. This study was deemed to be exempt as per our Institutional review board. Patients with AJCC stage I and II oral tongue cancer diagnosed between 2006 and 2013 were queried. Inclusion criteria entailed oral cavity tumors (tongue) with wide excision, stage I and II, histology codes 8052, 8070-8078, and 8083. Patients receiving chemotherapy, had an OS less than six months, underwent any RT other than EBRT, or any residual tumor after surgical resection, were excluded from analysis.

Patients were stratified by receipt of PORT, elective neck dissection, and extent of tumor DOI ( $\leqslant 4 \mathrm{~mm}$ or $>4$ $\mathrm{mm}$ ). Kaplan-Meier analysis was performed to compare OS (using the log-rank test) between patients receiving and not receiving PORT. Multivariable Cox proportional hazards regression model was used to evaluate the independent effect of PORT on OS, while controlling for tumor DOI and other clinical characteristics. The patient inclusion flow diagram is shown in Figure 1.

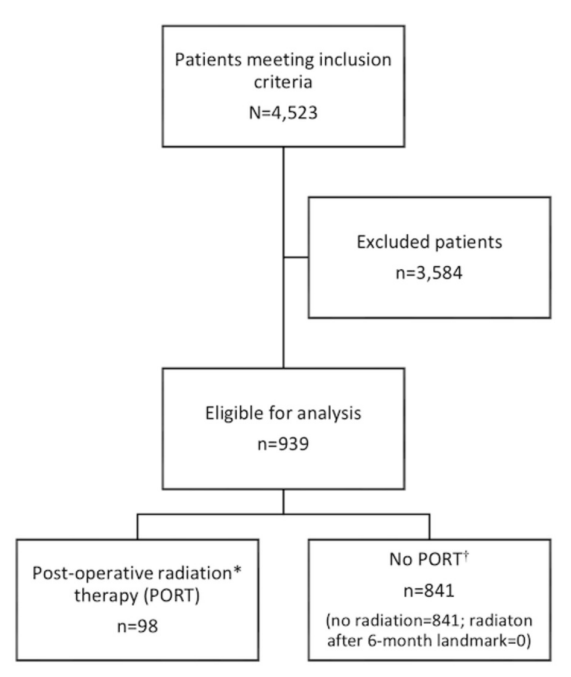

FIGURE 1: Patient inclusion flow diagram.

\section{Results}

Patient characteristics are summarized in Table 1 and comparisons of patients in the $<4 \mathrm{~mm}$ DOI and $>4 \mathrm{~mm}$ DOI groups are shown in Tables 2, 3. Ninety-eight (10.5\%) patients received RT while 841 (89.5\%) did not. Overall, a greater percentage of patients did not receive PORT. This trend persevered among each tumor stage. Additionally, African Americans were less likely to undergo PORT. In addition, the patients not receiving PORT were more likely to have $\mathrm{LVI}$ in the $<4 \mathrm{~mm}$ category. 


\section{Cureus}

\begin{tabular}{|c|c|c|c|c|c|}
\hline & PORT & & & No PORT & \\
\hline Characteristic & $\mathrm{N}$ & $\begin{array}{l}\text { Mean (SD) or } \\
\text { Freq. }(\%)\end{array}$ & $\mathrm{N}$ & $\begin{array}{l}\text { Mean (SD) or } \\
\text { Freq. (\%) }\end{array}$ & $\begin{array}{l}\mathrm{p}- \\
\text { value }^{*}\end{array}$ \\
\hline Age at Diagnosis & 98 & $57.8(14.0)$ & 841 & $59.5(15.0)$ & 0.346 \\
\hline Sex & 98 & & 841 & & \\
\hline Male & 57 & $58.2 \%$ & 443 & $52.7 \%$ & 0.303 \\
\hline Female & 41 & $41.8 \%$ & 398 & $47.3 \%$ & \\
\hline Race & 98 & & 841 & & \\
\hline Black & 7 & $7.1 \%$ & 15 & $1.8 \%$ & $0.015^{\dagger}$ \\
\hline White & 84 & $85.7 \%$ & 780 & $92.8 \%$ & \\
\hline Others & 6 & $6.1 \%$ & 36 & $4.3 \%$ & \\
\hline Unknown & 1 & $1.0 \%$ & 10 & $1.2 \%$ & \\
\hline Time from Diagnosis to Treatment (Days) & 98 & $26.2(19.0)$ & 803 & $28.5(31.9)$ & 0.865 \\
\hline Time from Diagnosis to Surgery (Days) & 98 & $28.1(19.3)$ & 803 & $33.9(32.1)$ & 0.124 \\
\hline Time from Diagnosis to Radiation Therapy (Days) & 98 & $79.0(27.8)$ & 0 & -- & -- \\
\hline Tumor Grade & 98 & & 841 & & \\
\hline Well differentiated, differentiated, NOS & 23 & $23.5 \%$ & 275 & $32.7 \%$ & 0.124 \\
\hline $\begin{array}{l}\text { Moderately differentiated, moderately well differentiated, intermediate } \\
\text { differentiation }\end{array}$ & 56 & $57.1 \%$ & 435 & $51.7 \%$ & \\
\hline Poorly differentiated & 15 & $15.3 \%$ & 83 & $9.9 \%$ & \\
\hline $\begin{array}{l}\text { Cell type not determined, not stated or not applicable, unknown } \\
\text { primaries, high-grade dysplasia }\end{array}$ & 4 & $4.1 \%$ & 48 & $5.7 \%$ & \\
\hline Clinical Stage & 98 & & 841 & & \\
\hline Stage I & 39 & $39.8 \%$ & 612 & $72.8 \%$ & $<0.001$ \\
\hline Stage II & 59 & $60.2 \%$ & 229 & $27.2 \%$ & \\
\hline Pathological Stage & 98 & & 841 & & \\
\hline Stage I & 50 & $51.0 \%$ & 670 & $79.7 \%$ & $<0.001$ \\
\hline Stage II & 48 & $49.0 \%$ & 171 & $20.3 \%$ & \\
\hline Analytic Stage & 98 & & 841 & & \\
\hline Stage I & 50 & $51.0 \%$ & 670 & $79.7 \%$ & $<0.001$ \\
\hline Stage II & 48 & $49.0 \%$ & 171 & $20.3 \%$ & \\
\hline Tumor Depth & 98 & & 841 & & \\
\hline$\leq 4 \mathrm{~mm}$ & 62 & $63.3 \%$ & 657 & $78.1 \%$ & 0.001 \\
\hline$>4 \mathrm{~mm}$ & 36 & $36.7 \%$ & 184 & $21.9 \%$ & \\
\hline Regional Lymph Node Surgery & 98 & & 841 & & \\
\hline Yes & 80 & $81.6 \%$ & 553 & $65.8 \%$ & 0.002 \\
\hline No & 18 & $18.4 \%$ & 288 & $34.2 \%$ & \\
\hline Lymph Vascular Invasion & 93 & & 813 & & \\
\hline Present & 12 & $12.9 \%$ & 31 & $3.8 \%$ & $0.006^{\dagger}$ \\
\hline Not present & 70 & $75.3 \%$ & 681 & $83.8 \%$ & \\
\hline Not applicable & 1 & $1.1 \%$ & 9 & $1.1 \%$ & \\
\hline
\end{tabular}




\section{Cureus}

Unknown

10

$10.8 \%$

$92 \quad 11.3 \%$

\section{TABLE 1: Patient characteristics by post-operative radiation therapy (PORT) status.}

*All continuous variables were analyzed using the Wilcoxon rank sum test, and all categorical variables were analyzed using the Chi-squared test, except those denoted with $t$, in which Fisher's exact test was used.

\begin{tabular}{|c|c|c|c|c|c|}
\hline & PORT & & & No PORT & \\
\hline Characteristic & $\mathrm{N}$ & $\begin{array}{l}\text { Mean (SD) or } \\
\text { Freq. (\%) }\end{array}$ & $\mathrm{N}$ & $\begin{array}{l}\text { Mean (SD) or } \\
\text { Freq. (\%) }\end{array}$ & $\begin{array}{l}\text { p- } \\
\text { value }^{*}\end{array}$ \\
\hline Age at Diagnosis & 62 & $56.9(14.0)$ & 657 & $59.6(15.1)$ & 0.158 \\
\hline Sex & 62 & & 657 & & \\
\hline Male & 35 & $56.5 \%$ & 343 & $52.2 \%$ & 0.522 \\
\hline Female & 27 & $43.6 \%$ & 314 & $47.8 \%$ & \\
\hline Race & 62 & & 657 & & \\
\hline Black & 3 & $4.8 \%$ & 11 & $1.7 \%$ & $0.273^{\dagger}$ \\
\hline White & 56 & $90.3 \%$ & 609 & $92.7 \%$ & \\
\hline Others & 2 & $3.2 \%$ & 29 & $4.4 \%$ & \\
\hline Unknown & 1 & $1.6 \%$ & 8 & $1.2 \%$ & \\
\hline Time from Diagnosis to Treatment (Days) & 62 & $28.0(19.9)$ & 657 & $28.9(34.4)$ & 0.435 \\
\hline Time from Diagnosis to Surgery (Days) & 62 & $29.3(18.8)$ & 657 & 34.9 (34.6) & 0.360 \\
\hline Time from Diagnosis to Radiation Therapy (Days) & 62 & 78.4 (28.2) & 0 & -- & -- \\
\hline Tumor Grade & 62 & & 657 & & \\
\hline Well differentiated, differentiated, NOS & 16 & $25.8 \%$ & 233 & $35.5 \%$ & 0.078 \\
\hline $\begin{array}{l}\text { Moderately differentiated, moderately well differentiated, intermediate } \\
\text { differentiation }\end{array}$ & 33 & $53.2 \%$ & 320 & $48.7 \%$ & \\
\hline Poorly differentiated & 11 & $17.7 \%$ & 61 & $9.3 \%$ & \\
\hline $\begin{array}{l}\text { Cell type not determined, not stated or not applicable, unknown } \\
\text { primaries, high-grade dysplasia }\end{array}$ & 2 & $3.2 \%$ & 43 & $6.5 \%$ & \\
\hline Clinical Stage & 62 & & 657 & & \\
\hline Stage I & 25 & $40.3 \%$ & 502 & $76.4 \%$ & $<0.001$ \\
\hline Stage II & 37 & $59.7 \%$ & 155 & $23.6 \%$ & \\
\hline Pathological Stage & 62 & & 657 & & \\
\hline Stage I & 33 & $53.2 \%$ & 538 & $81.9 \%$ & $<0.001$ \\
\hline Stage II & 29 & $46.8 \%$ & 119 & $18.1 \%$ & \\
\hline Analytic Stage & 62 & & 657 & & \\
\hline Stage I & 33 & $53.2 \%$ & 538 & $81.9 \%$ & $<0.001$ \\
\hline Stage II & 29 & $46.8 \%$ & 119 & $18.1 \%$ & \\
\hline Regional Lymph Node Surgery & 62 & & 657 & & \\
\hline Yes & 52 & $83.9 \%$ & 402 & $61.2 \%$ & $<0.001$ \\
\hline No & 10 & $16.1 \%$ & 255 & $38.8 \%$ & \\
\hline
\end{tabular}




\section{Cureus}

\begin{tabular}{llllll} 
Lymph Vascular Invasion & 58 & \multicolumn{5}{c}{631} \\
Present & 8 & $13.8 \%$ & 19 & $3.0 \%$ & $0.005^{\dagger}$ \\
Not present & 44 & $75.9 \%$ & 528 & $83.7 \%$ & \\
Not applicable & 1 & $1.7 \%$ & 9 & $1.4 \%$ & \\
Unknown & 5 & $8.6 \%$ & 75 & $11.9 \%$
\end{tabular}

TABLE 2: Characteristics of patients with tumor depth $\leq 4 \mathrm{~mm}$ by post-operative radiation therapy (PORT) status.

*All continuous variables were analyzed using the Wilcoxon rank sum test, and all categorical variables were analyzed using the Chi-squared test, except those denoted with $\dagger$, in which Fisher's exact test was used.

\begin{tabular}{|c|c|c|c|c|c|}
\hline \multirow[b]{2}{*}{ Characteristic } & \multicolumn{2}{|c|}{ PORT } & \multirow[b]{2}{*}{$\mathrm{N}$} & \multirow{2}{*}{$\begin{array}{l}\text { No PORT } \\
\text { Mean (SD) or } \\
\text { Freq. (\%) }\end{array}$} & \multirow[b]{2}{*}{$\begin{array}{l}\mathrm{p}- \\
\text { value }^{*}\end{array}$} \\
\hline & $\mathrm{N}$ & $\begin{array}{l}\text { Mean (SD) or } \\
\text { Freq. (\%) }\end{array}$ & & & \\
\hline Age at Diagnosis & 36 & $59.3(14.1)$ & 184 & $59.1(14.8)$ & 0.704 \\
\hline Sex & 36 & & 184 & & \\
\hline Male & 22 & $61.1 \%$ & 100 & $54.4 \%$ & 0.455 \\
\hline Female & 14 & $38.9 \%$ & 84 & $45.7 \%$ & \\
\hline Race & 36 & & 184 & & \\
\hline Black & 4 & $11.1 \%$ & 4 & $2.2 \%$ & 0.014 \\
\hline White & 28 & $77.8 \%$ & 171 & $92.9 \%$ & \\
\hline Others & 4 & $11.1 \%$ & 7 & $3.8 \%$ & \\
\hline Unknown & 0 & $0 \%$ & 2 & $1.1 \%$ & \\
\hline Time from Diagnosis to Treatment (Days) & 36 & $23.2(17.3)$ & 175 & $26.9(20.5)$ & 0.397 \\
\hline Time from Diagnosis to Surgery (Days) & 36 & $26.0(20.2)$ & 175 & $30.0(20.9)$ & 0.284 \\
\hline Time from Diagnosis to Radiation Therapy (Days) & 36 & $79.9(27.5)$ & 0 & -- & -- \\
\hline Tumor Grade & 36 & & 184 & & \\
\hline Well differentiated, differentiated, NOS & 7 & $19.4 \%$ & 42 & $22.8 \%$ & $0.763^{\top}$ \\
\hline $\begin{array}{l}\text { Moderately differentiated, moderately well differentiated, intermediate } \\
\text { differentiation }\end{array}$ & 23 & $63.9 \%$ & 115 & $62.5 \%$ & \\
\hline Poorly differentiated & 4 & $11.1 \%$ & 22 & $12.0 \%$ & \\
\hline $\begin{array}{l}\text { Cell type not determined, not stated or not applicable, unknown } \\
\text { primaries, high-grade dysplasia }\end{array}$ & 2 & $5.6 \%$ & 5 & $2.7 \%$ & \\
\hline Clinical Stage & 36 & & 184 & & \\
\hline Stage I & 14 & $38.9 \%$ & 110 & $59.8 \%$ & 0.021 \\
\hline Stage II & 22 & $61.1 \%$ & 74 & $40.2 \%$ & \\
\hline Pathological Stage & 36 & & 184 & & \\
\hline Stage I & 17 & $47.2 \%$ & 132 & $71.7 \%$ & 0.004 \\
\hline Stage II & 19 & $52.8 \%$ & 52 & $28.3 \%$ & \\
\hline Analytic Stage & 36 & & 184 & & \\
\hline Stage I & 17 & $47.2 \%$ & 132 & $71.7 \%$ & 0.004 \\
\hline
\end{tabular}




\section{Cureus}

\begin{tabular}{lllll} 
Stage II & 19 & $52.8 \%$ & 52 & $28.3 \%$ \\
Regional Lymph Node Surgery & 36 & & 184 & \\
Yes & 28 & $77.8 \%$ & $15182.1 \%$ & 0.546 \\
No & 8 & $22.2 \%$ & 33 & $17.9 \%$ \\
Lymph Vascular Invasion & 35 & & 182 & -- \\
Present & 4 & $11.4 \%$ & $12 \quad 6.6 \%$ & $15384.1 \%$ \\
Not present & 26 & $74.3 \%$ & 0 & \\
Not applicable & 0 & $0 \%$ & $17 \%$ & $9.3 \%$ \\
Unknown & 5 & $14.3 \%$ & & \\
\hline
\end{tabular}

\section{TABLE 3: Characteristics of patients with tumor depth $>4 \mathrm{~mm}$ by post-operative radiation therapy}

(PORT) status.

*All continuous variables were analyzed using the Wilcoxon rank sum test, and all categorical variables were analyzed using the Chi-squared test, except those denoted with $\dagger$, in which Fisher's exact test was used.

For tumors $<4 \mathrm{~mm}$ DOI, adding RT did not improve survival $(\mathrm{p}=0.634)$. OS was similar in patients with DOI $>4 \mathrm{~mm}$ with or without RT ( $\mathrm{p}=0.816$ ) (Figure 2$)$. Among those with tumor DOI $<4 \mathrm{~mm}$, clinical stage I patients trended towards improved OS compared to patients with clinical stage II tumors $(\mathrm{p}=0.07)$, and those with pathological stage I tumors trended towards improved OS in comparison to pathological stage II lesions ( $p=0.087)$. There was no difference in OS with respect to clinical stage $(p=0.445)$ (Figure 3$)$, and pathological stage $(\mathrm{p}=0.108)$ (Figure 4$)$ among patients with a tumor DOI $>4 \mathrm{~mm}$.

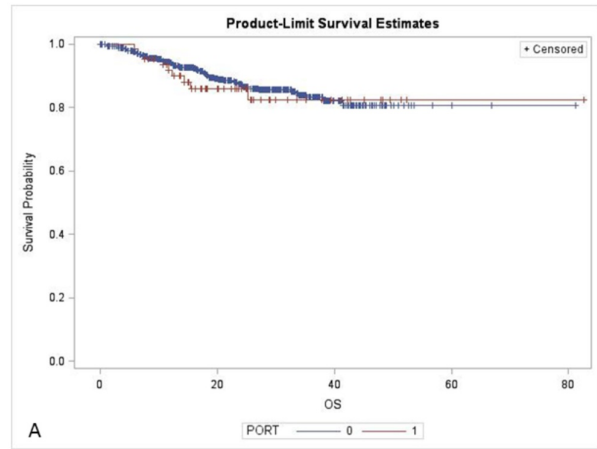

Log-Rank Test: $p=0.634$

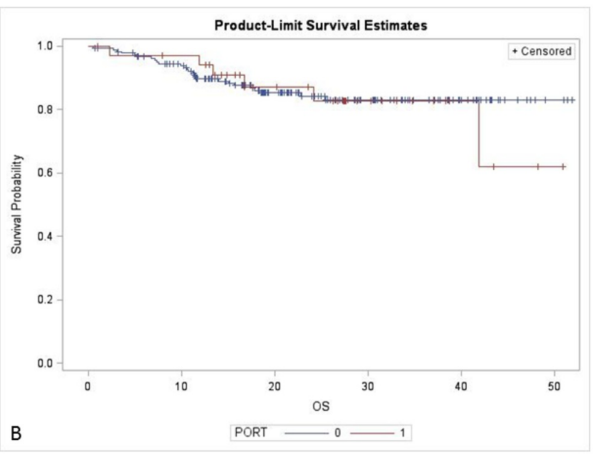

Log-Rank Test: $p=0.816$

FIGURE 2: Overall survival by PORT status with DOI: (A) $<4 \mathrm{~mm},(B)>4$ $\mathrm{mm}$.

PORT: Post-operative radiotherapy; DOI: Depth of invasion. 


\section{Cureus}

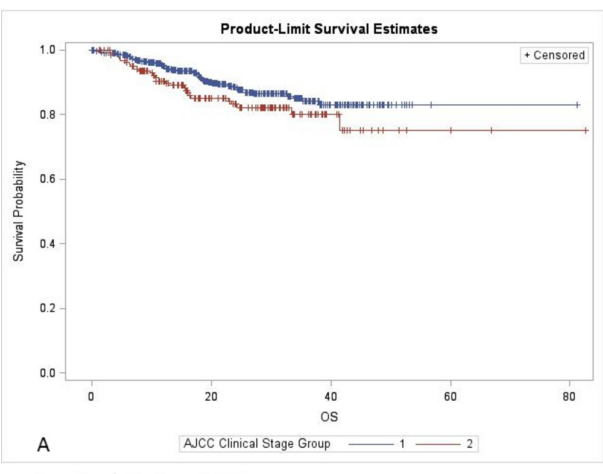

Log-Rank Test: $p=0.071$

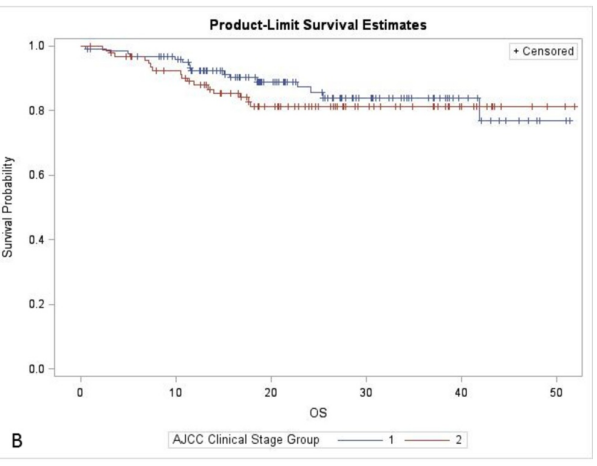

Log-Rank Test: $p=0.445$

FIGURE 3: Overall survival by clinical stage with DOI: (A) $<4 \mathrm{~mm}$, (B) $>4$ $\mathrm{mm}$.

DOI: Depth of invasion

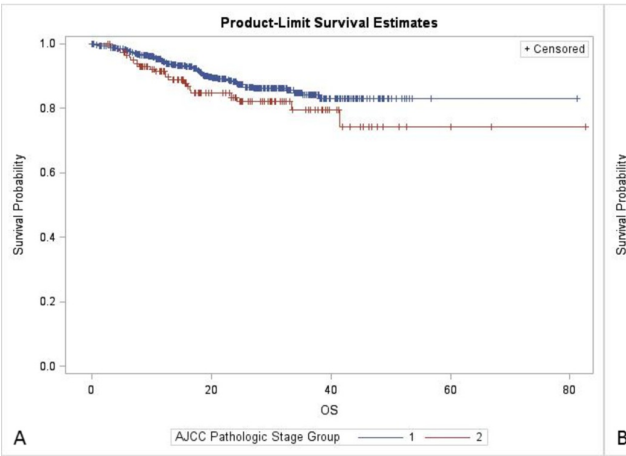

Log-Rank Test: $p=0.087$

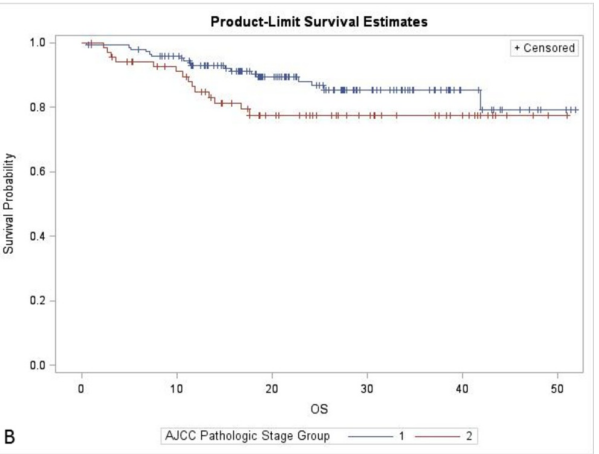

Log-Rank Test: $p=0.108$

FIGURE 4: Overall survival by pathological stage with DOI: (A) $<4 \mathrm{~mm}$, (B) $>4 \mathrm{~mm}$.

DOI: Depth of invasion

While elective neck dissection (END) did not impact OS for lesions with DOI <4 mm ( $\mathrm{p}=0.128)$, it did confer a survival benefit for lesions with DOI $>4 \mathrm{~mm}(\mathrm{p}=0.01)$ (Figure 5). 


\section{Cureus}

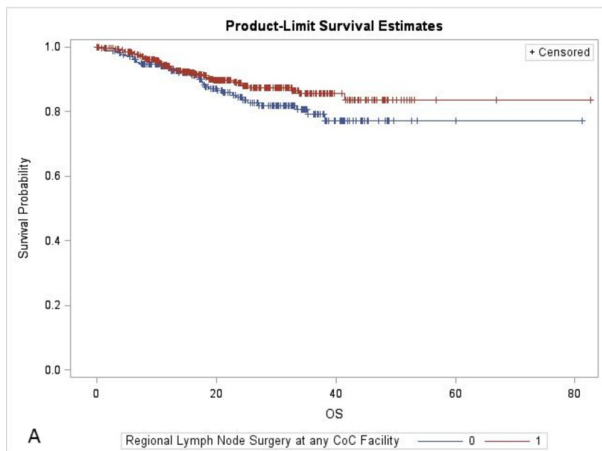

Log-Rank Test: $p=0.128$

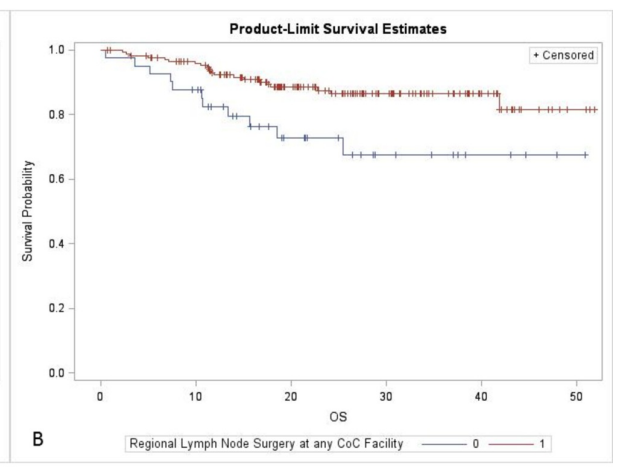

Log-Rank Test: $p=0.010$

FIGURE 5: Overall survival by elective neck surgery for tumors with DOI: (A) $<4 \mathrm{~mm}$, (B) $>4 \mathrm{~mm}$.

DOI: Depth of invasion

On multivariable survival analysis, END remained associated with an improved OS in the subset of patients with a DOI $>4 \mathrm{~mm}$ (hazard ratio of death, $0.37 ; 95 \%$ confidence interval, 0.17-0.81 [ $\mathrm{p}=0.012]$ ), when also controlling for age, sex, PORT status, clinical stage, and pathological stage (Tables 4, 5).

\section{Characteristic}

PORT

No

Yes

Age

Sex

Male

Female

Clinical Stage

Stage I

Stage II

Pathological Stage

Stage I

Stage II

Regional Lymph Node Surgery

No

Yes
Hazard Ratio (95\% Cl)

p-Value

$1.11(0.54,2.27)$

0.782

$1.03(1.01,1.05)$

$<0.001$

$0.84(0.54,1.30)$

0.425

$1.55(0.83,2.90)$

0.172

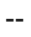

$1.27(0.66,2.45)$

0.482

$0.72(0.45,1.14)$

TABLE 4: Multivariable Cox regression model for overall survival (OS) tumor depth $\leq 4 \mathrm{~mm}$. 


\section{Cureus}

\begin{tabular}{|l|l|l|}
\hline Characteristic & Hazard Ratio $(95 \%$ Cl) & p-Value \\
\hline PORT & -- & -- \\
No & $0.80(0.32,2.01)$ & 0.641 \\
Yes & $1.04(1.01,1.07)$ & 0.011 \\
Age & & -- \\
Sex & -- & 0.319 \\
Male & $0.69(0.33,1.44)$ & -- \\
Female & & 0.943 \\
Clinical Stage & -- & - \\
Stage I & $1.03(0.42,2.57)$ & 0.129 \\
Stage II & & - \\
Pathological Stage & -- & \\
Stage I & $2.00(0.82,4.89)$ & \\
Stage II & -- & \\
Regional Lymph Node Surgery & $0.37(0.17,0.81)$ & \\
No & & \\
Yes & & \\
\hline
\end{tabular}

TABLE 5: Multivariable Cox regression model for overall survival (OS) tumor depth $>4 \mathrm{~mm}$.

PORT: Post-operative radiotherapy

\section{Discussion}

This population-based study of stage I and II oral tongue cancers showed a survival benefit of elective neck dissection in patients with DOI $>4 \mathrm{~mm}$, but no benefit of adding adjuvant RT in regard of less of DOI.

Numerous studies identify DOI as a poor prognostic factor. A retrospective Japanese study of 337 stage I-II tongue cancer patients undergoing surgical resection revealed that T stage, DOI (cut-off was $4 \mathrm{~mm}$ ), tumor budding (the presence of a single cancer cell or cluster of less than five cancer cells at the invasive front) and adjacent tissue at the invasive front are predictive of delayed neck metastasis [11].

Although $4 \mathrm{~mm}$ is commonly considered the DOI cut off for significance, a retrospective study of DOI cut-off points in previously untreated early stage oral tongue cancers showed $7.25 \mathrm{~mm}$ to be most predictive of occult nodal metastasis, $8 \mathrm{~mm}$ for OS and DFS [3]. In another retrospective study of 93 early stage oral lung cancer patients undergoing primary resection without neck dissection, $47.4 \%$ had nodal recurrence, with $19.7 \%$ recurred at the primary site. Cox-proportional polynomial analysis showed an increasing hazard of recurrence with DOI between 2-6 $\mathrm{mm}[4]$.

Ganly et al. sought to determine factors associated with tumor recurrence in a cohort of 216 patients with oral tongue cancers. Half of the lesions were T2, 83\% underwent surgery and $17 \%$ underwent surgery and PORT. At a median follow-up of 80 months, MVA revealed DOI as an independent predictor of neck relapsefree survival, with a DOI $>2 \mathrm{~mm}$ conferring 3.7-fold higher risk of recurrence compared to DOI $<2 \mathrm{~mm}[12]$.

A retrospective review evaluated outcomes of 103 patients with T1 or T2 N0 oral tongue cancers who underwent surgical resection with negative margins and DOI $>4 \mathrm{~mm}$. Sixty-two patients received PORT and 41 did not. With a median follow-up of 41.3 months, there was no difference between PORT versus no PORT [13].

Shim et al. reviewed the medical records of 86 patients with oral tongue cancers, of which $58 \%$ were stage I, $26 \%$ stage II and 16\% stage III. Among the 16\% receiving PORT, they reported no difference in recurrence rates for tumors $>0.5 \mathrm{~cm}$ compared to those who did not receive PORT [14]. Table 6 summarizes select studies evaluating DOI as a prognostic factor. 


\section{Cureus}

\begin{tabular}{|c|c|c|c|c|}
\hline $\begin{array}{l}\text { Author, } \\
\text { year } \\
\text { (reference) }\end{array}$ & Study design & $\begin{array}{l}\text { Significant } \\
\text { DOI }\end{array}$ & Outcomes & Comments \\
\hline $\begin{array}{l}\text { Fukano et } \\
\text { al., } 1997 \\
{[15]}\end{array}$ & $\begin{array}{l}\text { Retrospective, } 34 \text { patients, oral } \\
\text { tongue cancer }\end{array}$ & $5 \mathrm{~mm}$ & $>5 \mathrm{~mm}$, neck metastasis $64.7 \%$ & $\begin{array}{l}\text { For } \mathrm{DOI}>5 \mathrm{~mm} \text {, suggestion } \\
\text { is to operate or radiate neck }\end{array}$ \\
\hline $\begin{array}{l}\text { Asakage et } \\
\text { al., } 1998 \\
{[16]}\end{array}$ & $\begin{array}{l}\text { Retrospective, } 44 \text { patients, oral } \\
\text { tongue, stage I/II partial } \\
\text { glossectomy only }\end{array}$ & $4 \mathrm{~mm}$ & $\begin{array}{l}\text { Cervical metastasis in } 21 / 44 \text { patients, } \\
>4 \mathrm{~mm} \text { only factor significant in MVA }\end{array}$ & $\begin{array}{l}\text { Recommended } \\
\text { supraomohyoid neck } \\
\text { dissection in tumors }>4 \mathrm{~mm} \text {. }\end{array}$ \\
\hline $\begin{array}{l}\text { Kurokawa } \\
\text { et al., } 2002 \\
{[17]}\end{array}$ & $\begin{array}{l}\text { Retrospective, } 50 \text { patients, } \\
\text { stage I/II oral tongue, only } \\
\text { partial glossectomy }\end{array}$ & $4 \mathrm{~mm}$ & $\begin{array}{l}\text { Overall cervical metastasis rate of } 14 \% \text {, } \\
\text { MVA showed } \mathrm{DOI}>4 \mathrm{~mm} \text { as the } \\
\text { significant risk factor }\end{array}$ & $\begin{array}{l}\text { Recommended to electively } \\
\text { treat the neck for } \mathrm{DOI}>4 \mathrm{~mm}\end{array}$ \\
\hline $\begin{array}{l}\text { Goodman } \\
\text { et al., } 2009 \\
{[18]}\end{array}$ & $\begin{array}{l}\text { SEER, DOI, LVI and PNI } \\
\text { assessed with respect to } \\
\text { mortality }\end{array}$ & $3 \mathrm{~mm}$ & $\begin{array}{l}\text { MVI showed DOI and PNI were } \\
\text { significant predictors of OS }\end{array}$ & \\
\hline $\begin{array}{l}\text { Ling et al., } \\
2013 \text { [19] }\end{array}$ & $\begin{array}{l}\text { Retrospective, } 210 \text { patients with } \\
\text { tongue cancer }\end{array}$ & $9 \mathrm{~mm}$ & $\begin{array}{l}\text { DOI }>9 \mathrm{~mm} 7.7 \text { times more likely to die } \\
\text { than tumors }<4 \mathrm{~mm}\end{array}$ & $\begin{array}{l}\text { To improve survival in such } \\
\text { patients, surgical resection } \\
\text { recommended. }\end{array}$ \\
\hline $\begin{array}{l}\text { Almangush } \\
\text { et al., } 2014 \\
\text { [20] }\end{array}$ & $\begin{array}{l}\text { Retrospective study of } 233 \\
\text { patients with stage I/II oral } \\
\text { tongue cancers }\end{array}$ & $4 \mathrm{~mm}$ & $\begin{array}{l}\text { Tumor budding and } \mathrm{DOI}>4 \mathrm{~mm} \\
\text { associated with worse prognosis }\end{array}$ & $\begin{array}{l}\text { Recommended multimodality } \\
\text { therapy for deep tumors. }\end{array}$ \\
\hline $\begin{array}{l}\text { Masood et } \\
\text { al., } 2018 \\
{[21]}\end{array}$ & $\begin{array}{l}\text { Retrospective study, } 67 \text { patients } \\
\text { with T1/2N0 oral tongue cancer } \\
\text { HPV- }\end{array}$ & $5 \mathrm{~mm}$ & $\begin{array}{l}\mathrm{DOI}>5 \mathrm{~mm} \text { associated with risk of LVI } \\
\text { and nodal metastasis }\end{array}$ & $\begin{array}{l}\text { No specific recommendation } \\
\text { made regarding } \\
\text { management. }\end{array}$ \\
\hline
\end{tabular}

TABLE 6: Select studies evaluating DOI as a prognostic factor.

DOI: Depth of invasion; MVA: Multivariate analysis; LVI: Lympho-vascular invasion; PNI: Perineural invasion; HPV: Human papillomavirus.

Limitations of our study include its retrospective nature, relatively small number in the total group receiving $\mathrm{RT}$ and lack of data on details of treatment such as technique of RT, use of image guidance and dose, local control and toxicity. Select studies evaluating DOI as a prognostic factor are listed in Table 6. In clinical practice, DOI does dictate neck dissection based on risk of neck metastasis although we show survival benefit with END in DOI $>4 \mathrm{~mm}$. RT is associated with significant side effects including mucositis, pain, dysphagia, necrosis, dry mouth and loss of taste, and can be avoided for early stage tongue cancers.

\section{Conclusions}

Our study is the first large population-based study of both stage I and II oral cavity cancers to show addition of elective neck irradiation for tumors $>4 \mathrm{~mm}$ does not improve survival. However, elective neck dissection in oral tongue cancers with DOI $>4 \mathrm{~mm}$ confers a positive survival benefit.

\section{Additional Information}

\section{Disclosures}

Human subjects: Consent was obtained by all participants in this study. Animal subjects: All authors have confirmed that this study did not involve animal subjects or tissue. Conflicts of interest: In compliance with the ICMJE uniform disclosure form, all authors declare the following: Payment/services info: All authors have declared that no financial support was received from any organization for the submitted work. Financial relationships: All authors have declared that they have no financial relationships at present or within the previous three years with any organizations that might have an interest in the submitted work. Other relationships: All authors have declared that there are no other relationships or activities that could appear to have influenced the submitted work.

\section{References}

1. Jerjes W, Upile T, Petrie A, et al.: Clinicopathological parameters, recurrence, locoregional and distant metastasis in 115 T1-T2 oral squamous cell carcinoma patients. Head Neck Oncol. 2010, 2:9. 10.1186/17583284-2-9 
2. Huang SH, Hwang D, Lockwood G, Goldstein DP, O'Sullivan B: Predictive value of tumor thickness for cervical lymph-node involvement in squamous cell carcinoma of the oral cavity: a meta-analysis of reported studies. Cancer. 2009, 115:1489-1497. 10.1002/cncr.24161

3. Tam S, Amit M, Zafereo M, Bell D, Weber RS: Depth of invasion as a predictor of nodal disease and survival in patients with oral tongue squamous cell carcinoma. Head Neck. 2019, 41:177-184. 10.1002/hed.25506

4. Shinn JR, Wood CB, Colazo JM, Harrell FE Jr, Rohde SL, Mannion K: Cumulative incidence of neck recurrence with increasing depth of invasion. Oral Oncol. 2018, 87:36-42. 10.1016/j.oraloncology.2018.10.015

5. O'steen L, Amdur RJ, Morris CG, Hitchcock KE, Mendenhall WM: Challenging the requirement to treat the contralateral neck in cases with $>4 \mathrm{~mm}$ tumor thickness in patients receiving postoperative radiation therapy for squamous cell carcinoma of the oral tongue or floor of mouth. Am J Clin Oncol. 2019, 42:89-91. 10.1097/COC.0000000000000480

6. Faisal M, Abu Bakar M, Sarwar A, et al.: Depth of invasion (DOI) as a predictor of cervical nodal metastasis and local recurrence in early stage squamous cell carcinoma of oral tongue (ESSCOT). PLoS One. 2018, 13:e0202632. 10.1371/journal.pone.0202632

7. Chang B, He W, Ouyang H, Peng J, Shen L, Wang A, Wu P: A prognostic nomogram incorporating depth of tumor invasion to predict long-term overall survival for tongue squamous cell carcinoma with R0 resection. J Cancer. 2018, 9:2107-2115. 10.7150/jca.24530

8. Rajappa SK, Ram D, Bhakuni YS, Jain A, Kumar R, Dewan AK: Survival benefits of adjuvant radiation in the management of early tongue cancer with depth of invasion as the indication. Head Neck. 2018, 40:22632270. 10.1002/hed.25329

9. Rubin SJ, Gurary EB, Qureshi MM, Salama AR, Ezzat WH, Jalisi S, Truong MT: Stage II oral tongue cancer: survival impact of adjuvant radiation based on depth of invasion. Otolaryngol Head Neck Surg. 2019, 160:77-84. 10.1177/0194599818779907

10. National cancer database. (2017). Accessed: August 31, 2017: https://www.facs.org/quality-pro grams/cancer/ncdb.

11. Yamakawa N, Kirita T, Umeda M, et al.: Tumor budding and adjacent tissue at the invasive front correlate with delayed neck metastasis in clinical early-stage tongue squamous cell carcinoma. J Surg Oncol. 2019, 119:370-378. 10.1002/jso.25334

12. Ganly I, Patel S, Shah J: Early stage squamous cell cancer of the oral tongue--clinicopathologic features affecting outcome. Cancer. 2012, 118:101-111. 10.1002/cncr.26229

13. Gokavarapu S, Parvataneni N, Rao S LM, Reddy R, Raju KV, Chander R: Role of postoperative radiation therapy (PORT) in pT1-T2 N0 deep tongue cancers. Oral Surg Oral Med Oral Pathol Oral Radiol. 2015, 120:227-231. 10.1016/j.0000.2015.08.002

14. Shim SJ, Cha J, Koom WS, Kim GE, Lee CG, Choi EC, Keum KC: Clinical outcomes for T1-2N0-1 oral tongue cancer patients underwent surgery with and without postoperative radiotherapy. Radiat Oncol. 2010, 5:43. 10.1186/1748-717X-5-43

15. Fukano H, Matsuura H, Hasegawa Y, Nakamura S: Depth of invasion as a predictive factor for cervical lymph node metastasis in tongue carcinoma. Head Neck. 1997, 19:205-210. 10.1002/(SICI)10970347(199705)19:3<205::AID-HED7>3.0.CO;2-6

16. Asakage T, Yokose T, Mukai K, Tsugane S, Tsubono Y, Asai M, Ebihara S: Tumor thickness predicts cervical metastasis in patients with stage I/II carcinoma of the tongue. Cancer. 1998, 82:1443-1448. 10.1002/(sici)1097-0142(19980415)82:8<1443::aid-cncr2>3.0.c0;2-a

17. Kurokawa H, Yamashita Y, Takeda S, Zhang M, Fukuyama H, Takahashi T: Risk factors for late cervical lymph node metastases in patients with stage I or II carcinoma of the tongue. Head Neck. 2002, 24:731-736. 10.1002/hed.10130

18. Goodman M, Liu L, Ward K, et al.: Invasion characteristics of oral tongue cancer: frequency of reporting and effect on survival in a population-based study. Cancer. 2009, 115:4010-4020. 10.1002/cncr.24459

19. Ling W, Mijiti A, Moming A: Survival pattern and prognostic factors of patients with squamous cell carcinoma of the tongue: a retrospective analysis of 210 cases. J Oral Maxillofac Surg. 2013, 71:775-785. 10.1016/j.joms.2012.09.026

20. Almangush A, Bello IO, Keski-Säntti H, et al.: Depth of invasion, tumor budding, and worst pattern of invasion: prognostic indicators in early-stage oral tongue cancer. Head Neck. 2014, 36:811-818. 10.1002/hed.23380

21. Masood MM, Farquhar DR, Vanleer JP, Patel SN, Hackman TG: Depth of invasion on pathological outcomes in clinical low-stage oral tongue cancer patients. Oral Dis. 2018, 24:1198-1203. 10.1111/odi.12887 